in vivo $32: 663-667(2018)$

doi:10.21873/invivo.11291

\title{
Systemic Immune-inflammation Index Predicts Survival of Patients After Curative Resection for Non-small Cell Lung Cancer
}

\author{
MASAKI TOMITA ${ }^{1}$, TAKANORI AYABE ${ }^{1}$, RYO MAEDA $^{1}$ and KUNIHIDE NAKAMURA ${ }^{2}$ \\ Departments of ${ }^{1}$ Thoracic and Breast Surgery, and ${ }^{2}$ Cardiovascular Surgery, \\ Faculty of Medicine, University of Miyazaki, Kiyotake, Japan
}

\begin{abstract}
Background: The systemic immune-inflammation index (SII) is reported to be associated with clinical outcomes and has been proven to be a promising prognostic indicator in several solid tumor types. To the best of our knowledge, however, no studies regarding SII in patients with resectable non-small cell lung cancer (NSCLC) are available. Materials and Methods: Three hundred forty-one patients with NSCLC who underwent surgery at our Institution between 2008 and 2012 were included. The SII was calculated using the formula: platelet count $\times$ neutrophil/lymphocyte count. The optimal cutoff value was calculated using the Cutoff Finder (http://molpath.charite.de/cutoff). Univariate and multivariate analyses were calculated by the Cox proportional hazards regression model. Results: The optimal cut-off value was $471.2 \times 109 / 1$ for SII. A low SII was associated with female gender, never smoking status, adenocarcinoma histology, higher pathological TNM stage and low level of serum $C$-reactive protein, but not age, serum carcinoembryonic antigen or cytokeratin 19 fragment level. Patients of the low SII group had a significantly better 5-year overall survival than those with high SII (83.61\% vs. $60.39 \%, p<0.001)$. Multivariate analysis revealed that the SII was a significant independent predictive indicator for cancer-specific survival $(p=0.007)$. Conclusion: This is the first study to demonstrate that the SII could represent an independent prognostic factor for patients with resectable NSCLC.
\end{abstract}

This article is freely accessible online.

Correspondence to: Masaki Tomita, MD, Department of Thoracic and Breast Surgery, Faculty of Medicine, University of Miyazaki, Kihara 5200, Kiyotake, Miyazaki, 889-1692, Japan. Tel: +81 985852291, Fax: +81 985855563, e-mail: mtomita@med.miyazakiu.ac.jp

Key Words: Systemic immune-inflammation index, non-small cell lung cancer, surgery, prognosis.
Cancer-related inflammation is an essential component of the tumor microenvironment, and inflammatory cells may play a critical role in tumor development and progression (1). It has been shown that markers of inflammation and scoring systems based on the systemic inflammatory response are of prognostic value in patients with non-small cell lung cancer (NSCLC) (2). In particular, various combinations of hematological parameters [including $\mathrm{C}$-reactive protein (CRP) concentration, the neutrophil-to-lymphocyte ratio (NLR), and the Glasgow prognostic score (GPS)] have been evaluated as predictors of recurrence or survival in NSCLC (3-6).

Recently, a novel index named the systemic immuneinflammation index (SII), based on the peripheral lymphocyte, neutrophil and platelet counts, was investigated as a prognostic marker in several tumor types (7). Geng et al. suggested that SII was superior to other such indices, such as NLR, and served as a more objective marker that reflects the balance between host inflammatory and immune response status in patients with esophageal squamous cell carcinoma (8).

With regard to lung cancer, the prognostic significance of SII was reported for small cell lung cancer (9) and stage III NSCLC (10). However, to our knowledge, there are no previous studies that evaluated the significance of SII for resectable NSCLC. Therefore, in the present study, we examined the prognostic significance of SII in patients with resectable NSCLC.

\section{Patients and Methods}

This retrospective study was approved by our Institutional Review Board (O-0255) and the need to obtain patient consent was waived. We retrospectively reviewed data from 341 patients (with 173 men and 168 women, median age of 69 years) who underwent complete resection for NSCLC at our hospital from January 2008 to December 2012. The inclusion criteria were as follows: histologically confirmed NSCLC and complete clinical, laboratory, imaging and follow-up data. The exclusion criteria included: preoperative chemotherapy/radiotherapy or death during the 
Table I. Clinical characteristics of patients based on the systemic immune-inflammation index (SII).

\begin{tabular}{|c|c|c|c|}
\hline \multirow[b]{2}{*}{ Characteristic } & \multicolumn{2}{|c|}{ SII $(\%)$} & \multirow[t]{2}{*}{$p$-Value } \\
\hline & Low & High & \\
\hline \multicolumn{4}{|l|}{ Age } \\
\hline$<65$ Years & $62(58.49)$ & $44(41.51)$ & \multirow[t]{2}{*}{0.276} \\
\hline$\geq 65$ Years & $152(64.68)$ & $83(35.32)$ & \\
\hline \multicolumn{4}{|l|}{ Gender } \\
\hline Male & $93(53.76)$ & $80(46.24)$ & \multirow[t]{2}{*}{0.001} \\
\hline Female & $121(72.02)$ & $47(27.98)$ & \\
\hline \multicolumn{4}{|l|}{ Smoking status } \\
\hline Never & $114(53.27)$ & $44(34.65)$ & \multirow[t]{2}{*}{0.001} \\
\hline Current/former & $100(46.73)$ & $83(65.35)$ & \\
\hline \multicolumn{4}{|l|}{ Histology } \\
\hline Adenocarcinoma & $182(67.91)$ & $86(32.09)$ & \multirow[t]{2}{*}{$<0.001$} \\
\hline Other & $32(43.84)$ & $41(56.16)$ & \\
\hline \multicolumn{4}{|l|}{ pStage } \\
\hline I & $178(67.17)$ & $87(32.83)$ & \multirow[t]{2}{*}{0.002} \\
\hline II-III & $36(47.37)$ & $40(52.63)$ & \\
\hline \multicolumn{4}{|l|}{ pT status } \\
\hline pT1 & $157(66.81)$ & $78(33.19)$ & \multirow[t]{2}{*}{0.022} \\
\hline pT2-3 & $57(53.77)$ & $49(46.23)$ & \\
\hline \multicolumn{4}{|l|}{ pN status } \\
\hline pNo & $192(65.75)$ & $100(34.25)$ & \multirow[t]{2}{*}{0.006} \\
\hline pN1-2 & $22(44.9)$ & $27(55.1)$ & \\
\hline \multicolumn{4}{|l|}{ Serum CEA } \\
\hline Normal & $159(62.6)$ & $95(37.4)$ & \multirow[t]{2}{*}{0.918} \\
\hline High & $55(63.22)$ & $32(36.76)$ & \\
\hline \multicolumn{4}{|l|}{ CYFRA21-1 } \\
\hline Normal & $181(63.96)$ & $102(36.04)$ & \multirow[t]{2}{*}{0.314} \\
\hline High & $33(56.9)$ & $25(43.1)$ & \\
\hline \multicolumn{4}{|l|}{ CRP } \\
\hline Low & $167(69.01)$ & $75(30.99)$ & \multirow[t]{2}{*}{$<0.001$} \\
\hline High & $47(47.47)$ & $52(52.53)$ & \\
\hline
\end{tabular}

CEA: Carcinoembryonic antigen, CYFRA21-1: cytokeratin 19 fragments, CRP: C-reactive protein.

perioperative period, clinical evidence of infection or other bone marrow, hematological or autoimmune disease. The last follow-up was in December 2017. Disease in all patients in this study was staged according to the eighth edition cancer staging manual (11). The blood samples for this analysis were collected before surgery. Data on preoperative peripheral neutrophil, lymphocyte and platelet counts, serum levels of CRP, carcinoembryonic antigen (CEA) and cytokeratin 19 fragments (CYFRA21-1) were extracted from the medical records. The SII was defined as follows: SII= platelet count $\times$ neutrophil/lymphocyte count. The optimal cut-off value of SII for overall survival was determined using the Cutoff Finder (http://molpath.charite.de/cutoff) (12).

Association between categorical variables was assessed using Fisher's exact test. The Kaplan-Meier method was used to estimate cancer-specific survival after surgery. The log-rank test and Cox proportional hazards regression were used to test for differences between groups. After univariate analysis, a multivariate analysis was carried out using Cox regression model. Estimated hazard

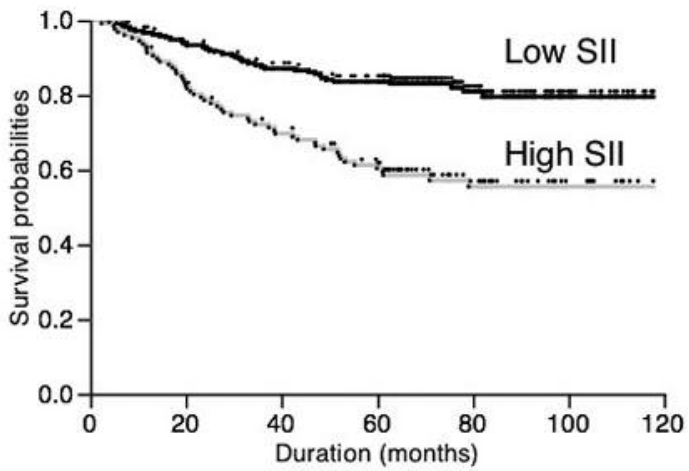

Figure 1. Overall postoperative cancer-specific survival of patients based on the systemic immune-inflammation index (SII). Patients were divided into two groups using the optimal cut-off value for SII of $471.2 \times 10^{9} / l$.

ratios, their $95 \%$ confidence intervals $(95 \% \mathrm{CI})$, and $p$-values were calculated from the Cox proportional hazards regression models. Statistical analyses were performed using JMP (SAS Institute Inc., Cary, NC, USA). Differences were considered statistically significant when $p<0.05$.

\section{Results}

The optimal cut-off value for SII was $471.2 \times 10^{9} / 1$ according to the Cutoff Finder. Patients then were divided into two groups using this cut-off. There were $214(62.76 \%)$ patients with low SII $\left(\leq 471.2 \times 10^{9} / 1\right)$ and $127(37.24 \%)$ patients with high SII $\left(>471.2 \times 10^{9} / 1\right)$. The relationships between SII and clinical characteristics are shown in Table I. In the present series, a low SII was significantly associated with female gender $(p=0.001)$, never smoking status $(p=0.001)$, adenocarcinoma histology $(p<0.001)$, lower pathological TNM stage $(p=0.002)$ and low serum CRP level $(p<0.001)$, but not age $(p=0.276)$, nor serum CEA $(p=0.918)$ or CYFRA21-1 $(p=0.314)$ levels.

The 5-year cancer-specific survival of patients with low SII was $83.61 \%$, while that of the high SII group was $60.39 \%(p<0.001)$. As shown in Figure 1, high SII was also associated with reduced overall cancer-specific survival.

On univariate Cox regression analysis, gender, smoking status, histology, pT status, pN status, serum CEA, CYFRA21-1 and CRP levels, and SII were significantly correlated with cancer-specific survival (all $p<0.001$, Table II). All nine clinicopathological characteristics were further investigated in multivariate analysis. As shown in Table III, gender $(p=0.002)$, histology $(p=0.001), \mathrm{pN}$ status $(p=0.003)$, serum CEA level $(p=0.001)$, CRP $(p=0.028)$ and SII $(p=0.007)$ were independent factors in predicting overall postoperative cancer-specific survival, while smoking status, pT status and serum CYFRA21-1 level were not. 
Table II. Univariate analysis of patients based on the systemic immune-inflammation index (SII).

\begin{tabular}{|c|c|c|c|c|c|c|}
\hline \multirow[t]{2}{*}{ Variable } & \multirow[t]{2}{*}{ Favorable } & \multirow[t]{2}{*}{ Unfavorable } & \multirow[t]{2}{*}{ Hazard ratio } & \multicolumn{2}{|c|}{ 95\% Confidence interval } & \multirow[t]{2}{*}{$p$-Value } \\
\hline & & & & Lower & Upper & \\
\hline Age & $<65$ Years & $\geq 65$ Years & 0.755 & 0.461 & 1.193 & 0.235 \\
\hline Gender & Female & Male & 0.329 & 0.204 & 0.514 & $<0.001$ \\
\hline Smoking status & Never & Current/former & 0.349 & 0.215 & 0.549 & $<0.001$ \\
\hline Histology & Adenocarcinoma & Other & 0.250 & 0.165 & 0.382 & $<0.001$ \\
\hline pT status & pT1 & pT2-3 & 0.450 & 0.298 & 0.683 & $<0.001$ \\
\hline $\mathrm{pN}$ status & pNO & $\mathrm{pN} 1-2$ & 0.330 & 0.211 & 0.534 & $<0.001$ \\
\hline CEA & Normal & High & 0.382 & 0.252 & 0.586 & $<0.001$ \\
\hline CYFRA21-1 & Normal & High & 0.353 & 0.229 & 0.556 & $<0.001$ \\
\hline CRP & Low & High & 0.393 & 0.260 & 0.594 & $<0.001$ \\
\hline SII & Low & High & 0.368 & 0.241 & 0.556 & $<0.001$ \\
\hline
\end{tabular}

CEA: Carcinoembryonic antigen, CYFRA21-1: cytokeratin 19 fragments, CRP: C-reactive protein.

Table III. Multivariate analysis of patients based on the systemic immune-inflammation index (SII).

\begin{tabular}{|c|c|c|c|c|c|c|c|}
\hline \multirow[t]{2}{*}{ Variable } & \multirow[t]{2}{*}{ Favorable } & \multirow[t]{2}{*}{ Unfavorable } & \multirow[t]{2}{*}{ Hazard ratio } & \multirow[t]{2}{*}{$95 \%$} & \multicolumn{2}{|c|}{ Confidence interval } & \multirow[t]{2}{*}{$p$-Value } \\
\hline & & & & & Lower & Upper & \\
\hline Gender & Female & Male & 0.358 & & 0.178 & 0.700 & 0.002 \\
\hline Smoking status & Never & Current/former & 0.687 & & 0.337 & 1.430 & 0.313 \\
\hline Histology & Adenocarcinoma & Other & 0.443 & & 0.275 & 0.720 & 0.001 \\
\hline pT status & pT1 & pT2-3 & 0.940 & & 0.572 & 1.526 & 0.804 \\
\hline pN status & $\mathrm{pN} 0$ & pN1-2 & 0.431 & & 0.255 & 0.743 & 0.003 \\
\hline CEA & Normal & High & 0.438 & & 0.280 & 0.694 & 0.001 \\
\hline CYFRA21-1 & Normal & High & 0.767 & & 0.465 & 1.295 & 0.315 \\
\hline CRP & Low & High & 0.604 & & 0.386 & 0.947 & 0.028 \\
\hline SII & Low & High & 0.540 & & 0.343 & 0.844 & 0.007 \\
\hline
\end{tabular}

CEA: Carcinoembryonic antigen, CYFRA21-1: cytokeratin 19 fragments, CRP: C-reactive protein.

\section{Discussion}

The link between inflammation and cancer has been widely investigated. Tumor cells influence expression of proinflammatory mediators, and systemic inflammation promotes tumor cell proliferation, migration and invasion by inhibiting apoptosis and promoting angiogenesis $(1,13,14)$. To the best of our knowledge, this is the first report to demonstrate the prognostic role of SII in patients with resectable NSCLC. In this study, we revealed that SII was an independent significant predictive factor $(p=0.007)$.

Several studies may give explanations for the prognostic value of SII in patients with tumors. Various studies have suggested that systemic immune and inflammatory cells, such as neutrophils, monocytes, platelets and lymphocytes, are associated with prognostic value in many malignancies (15-18).
Previous studies reported a prognostic significance of the NLR in NSCLC $(4,5)$. Lymphocytes play a fundamental role in cell-mediated immunological destruction of cancer cells (19). Furthermore, neutrophils have a crucial role in the pathogenesis of a broad range of diseases, including cancer (20). Therefore, the prognostic mechanism of the NLR is easy to understand.

Thrombocytosis is a paraneoplastic syndrome, and previous studies have found a significant role of platelets during tumor development and progression (21). Indeed, there is increasing evidence that a high platelet count is associated with worse survival in patients with NSCLC (22). Patients with a high SII often have thrombocytosis, neutrophilia, or lymphopenia. Thus, due to high numbers of neutrophils and platelets while having a low level of lymphocytes, a high SII usually indicates a stronger inflammatory and a weaker immune response in patients. It 
may be associated with invasion and metastasis of tumor cells and hence lead to poor survival. Thus, a high SII reflects alterations in the cancer microenvironment that favor cancer initiation, progression, and metastasis.

In the present series, a low SII was significantly associated with female gender, never smoking status, adenocarcinoma histology, lower pathological TNM stage and low CRP. This indicates that the SII is correlated with a more aggressive disease phenotype. However, the results of multivariable analysis showed that the prognostic significance of the SII was independent of standard characteristics of aggressive disease.

SII was based on the counts of three types of circulating immune cells, while NLR, GPS, platelet-to-lymphocyte ratio and monocyte-to-lymphocyte ratio, are based on two types. The SII should be a more objective marker that reflects the balance between host inflammatory and immune responses than all the other systemic inflammation scores. Geng et al. showed SII to be superior to the above indices as a prognostic predictive factor in patients with esophageal squamous cell carcinoma (8). A previous study showed that non-steroidal anti-inflammatory drugs have a role in the prevention and treatment of cancer (23) Therefore, there is a possibility that pw NSCLC who have a high SII may especially benefit from targeted anti-inflammatory with aspirin and non-steroidal anti-inflammatory drugs. In addition, the SII is simple and inexpensive to derive, and does not require extra equipment to that needed for day-today analyses. Thus, the SII should be routinely considered preoperatively for patients with NSCLC.

The present study had certain limitations. It was a retrospective, single-center study. Therefore, a large-scale prospective study is required to validate the results of the present study.

In conclusion, to our knowledge, this is the first study to demonstrate that the SII could represent an independent prognostic factor for patients with resectable NSCLC.

\section{Conflicts of Interest}

The Authors have declared that no conflict of interest exists in regard to this study.

\section{References}

1 Grivennikov SI, Greten FR and Karin M: Immunity, inflammation and cancer. Cell 140: 883-899, 2010.

2 Liao C, Yu Z, Guo W, Liu Q, Wu Y, Li Y and Bai L: Prognostic value of circulating inflammatory factors in non-small cell lung cancer: a systematic review and meta-analysis. Cancer Biomark 14: 469-481, 2014

3 Leuzzi G, Galeone C, Gisabella M, Duranti L, Taverna F, Suatoni P, Morelli D and Pastorino U: Baseline C-reactive protein level predicts survival of early-stage lung cancer: evidence from a systematic review and meta-analysis. Tumori 102: 441-449, 2016

4 Yin Y, Wang J, Wang X, Gu L, Pei H, Kuai S, Zhang Y and Shang Z: Prognostic value of the neutrophil to lymphocyte ratio in lung cancer: A meta-analysis. Clinics 70: 524-530, 2015.

$5 \mathrm{Gu}$ XB, Tian T, Tian XJ and Zhang XJ: Prognostic significance of neutrophil-to-lymphocyte ratio in non-small cell lung cancer: a meta-analysis. Sci Rep 5: 12493, 2015.

6 Tomita M, Ayabe T, Chosa E and Nakamura K: Prognostic significance of pre- and postoperative Glasgow prognostic score for patients with non-small cell lung cancer. Anticancer Res 34 : 3137-3140, 2014.

7 Hu B, Yang XR, Xu Y, Sun YF, Sun C, Guo W, Zhang X, Wang WM, Qiu SJ, Zhou J and Fan J: Systemic immune-inflammation index predicts prognosis of patients after curative resection for hepatocellular carcinoma. Clin Cancer Res 20: 6212-6222, 2014.

8 Geng Y, Shao Y, Zhu D, Zheng X, Zhou Q, Zhou W, Ni X, Wu $\mathrm{C}$ and Jiang J: Systemic immune-inflammation index predicts prognosis of patients with esophageal squamous cell carcinoma: a propensity score-matched analysis. Sci Rep 6: 39482, 2016.

9 Hong X, Cui B, Wang M, Yang Z, Wang L and Xu Q: Systemic immune-inflammation index, based on platelet counts and neutrophil-lymphocyte ratio, is useful for predicting prognosis in small cell lung cancer. Tohoku J Exp Med 236: 297-304, 2015.

10 Tong YS, Tan J, Zhou XL, Song YQ and Song YJ: Systemic immune-inflammation index predicting chemoradiation resistance and poor outcome in patients with stage III non-small cell lung cancer. J Transl Med 15: 221, 2017.

11 Detterbeck FC, Boffa DJ, Kim AW and Tanoue LT: The Eighth Edition Lung Cancer Stage Classification. Chest 151: 193-203, 2017.

12 Budczies J, Klauschen F, Sinn BV, Gyorffy B, Schmitt WD, Darb-Esfahani S and Denkert C: Cutoff Finder: a comprehensive and straightforward Web application enabling rapid biomarker cutoff optimization. PLoS One 7: e51862, 2012.

13 Shaverdian N, Wang J, Levin-Epstein R, Schaue D, Kupelian P, Lee P, Yang I, and Kaprealian T: Pro-inflammatory state portends poor outcomes with stereotactic radiosurgery for brain metastases. Anticancer Res 36: 5333-5337, 2016.

14 Mimatsu K, Fukino N, Ogasawara Y, Saino Y, and Oida T: Utility of inflammatory marker- and nutritional status-based prognostic factors for predicting the prognosis of stage iv gastric cancer patients undergoing non-curative surgery. Anticancer Res 37: 4215-4222, 2017.

15 Li TJ, Jiang YM, Hu YF, Huang L, Yu J, Zhao LY, Deng HJ, Mou TY, Liu H, Yang Y, Zhang Q and Li GX: Interleukin-17producing neutrophils link inflammatory stimuli to disease progression by promoting angiogenesis in gastric cancer. Clin Cancer Res 23: 1575-1585, 2017.

16 Eo WK, Jeong DW, Chang HJ, Won KY, Choi SI, Kim SH, Chun SW, Oh YL, Lee TH, Kim YO, Kim KH, Ji YI, Kim A and Kim HY: Absolute monocyte and lymphocyte count prognostic score for patients with gastric cancer. World J Gastroenterol 21: 2668-2676, 2015.

17 Kilincalp S, Ekiz F, Basar O, Ayte MR, Coban S, Yilmaz B, Altinbas A, Basar N, Aktas B, Tuna Y, Erbis H, Ucar E, Erarslan $\mathrm{E}$ and Yuksel O: Mean platelet volume could be possible biomarker in early diagnosis and monitoring of gastric cancer. Platelets 25: 592-594, 2014. 
18 Quigley DA and Kristensen V: Predicting prognosis and therapeutic response from interactions between lymphocytes and tumor cells. Mol Oncol 9: 2054-2062, 2015.

19 Ferrone $\mathrm{C}$ and Dranoff $\mathrm{G}$ : Dual roles for immunity in gastrointestinal cancers. J Clin Oncol 28: 4045-4051, 2010.

20 Mantovani A, Cassatella MA, Costantini C and Jaillon S: Neutrophils in the activation and regulation of innate and adaptive immunity. Nat Rev Immunol 11: 519-531, 2011.

21 Wojtukiewicz MZ, Sierko E, Hempel D, Tucker SC and Honn $\mathrm{KV}$ : Platelets and cancer angiogenesis nexus. Cancer Metastasis Rev 36: 249-262, 2017.
22 Tomita M, Shimizu T, Hara M, Ayabe T and Onitsuka T: Prognostic impact of thrombocytosis in resectable non-small cell lung cancer. Interact Cardiovasc Thorac Surg 7: 613-615, 2008.

23 Pereg D and Lishner M: Non-steroidal anti-inflammatory drugs for the prevention and treatment of cancer. J Intern Med 258: 115-123, 2005.

Received January 9, 2018

Revised February 13, 2018

Accepted February 20, 2018 